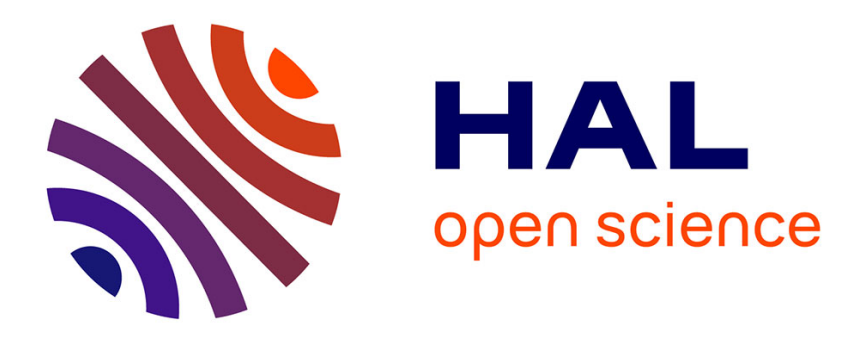

\title{
Semi-implicit two-speed Well-Balanced relaxation scheme for Ripa model
}

\author{
Emmanuel Franck, Laurent Navoret
}

\section{To cite this version:}

Emmanuel Franck, Laurent Navoret. Semi-implicit two-speed Well-Balanced relaxation scheme for Ripa model. FVCA 2020, Jun 2020, Bergen, Norway. pp 735-743, 10.1007/978-3-030-43651-3_70 . hal-02407820

\section{HAL Id: hal-02407820 \\ https://hal.science/hal-02407820}

Submitted on 12 Dec 2019

HAL is a multi-disciplinary open access archive for the deposit and dissemination of scientific research documents, whether they are published or not. The documents may come from teaching and research institutions in France or abroad, or from public or private research centers.
L'archive ouverte pluridisciplinaire HAL, est destinée au dépôt et à la diffusion de documents scientifiques de niveau recherche, publiés ou non, émanant des établissements d'enseignement et de recherche français ou étrangers, des laboratoires publics ou privés. 


\title{
Semi-implicit two-speed Well-Balanced relaxation scheme for Ripa model
}

\author{
Emmanuel Franck, Laurent Navoret
}

\begin{abstract}
In this paper, we propose a semi-implicit well-balanced scheme for the Ripa model based on a two-speed relaxation. The method both preserves equilibria and has an implicit step that reduces to the inversion of a constant Laplacian. Numerical simulations show that the scheme well capture low-Froude flows.
\end{abstract}

Key words: relaxation method, shallow-water model, semi-implicit scheme

\section{Introduction}

To discretize two-scale hyperbolic problems with a good accuracy, several methods have been proposed and are generally based on semi-implicit schemes to prevent us from the fast scale stringent stability condition. In [3, 5], such a scheme have been proposed and it is based on very recent relaxation method and a dynamical splitting. This method allows to adapt the time discretization to the regime and the implicit step just reduces to the inversion of a discrete Laplacian with constant coefficient. In this paper, we adapt this numerical scheme to the Ripa model with topography, which describes shallow-water flows with horizontal temperature gradients. In the Ripa model, the fast scale dynamics are perturbative gravity waves around an equilibrium and the slow scale dynamics is the convection. Here equilibria are balance between the pressure gradient and the topography source term. In addition to the implicit treatment of the perturbative waves, the scheme has to preserve the equilbria so to prevent the generation of spurious waves around them. This is the so-called wellbalanced property.

We consider the one-dimensional Ripa model [8]:

Emmanuel Franck, Laurent Navoret

INRIA Grand-Est and IRMA Strasbourg, 7 Rue René Descartes, 67000, Strasbourg, France, e-mail: emmanuel.franck@inria.fr, laurent.navoret@math.unistra.fr 


$$
\left\{\begin{array}{l}
\partial_{t} h+\partial_{x}(h u)=0 \\
\partial_{t}(h u)+\partial_{x}\left(h u^{2}+p(h, \Theta)\right)=-g h \Theta \partial_{x} z \\
\partial_{t}(h \Theta)+\partial_{x}(h \Theta u)=0
\end{array}\right.
$$

where $h(x, t)$ is the water height, $u(x, t)$ the velocity, $\Theta(x, t)$ the temperature and $z(x)$ the topography and the pressure law is given by: $p(h, \Theta)=g \Theta \frac{1}{2} h^{2}$, with $g$ the gravity constant. This system is hyperbolic and has three characteristic speeds: $\Sigma=\{u-c, u, u+c\}$, with $c=\sqrt{g h \Theta}$. We introduce the Froude number $\operatorname{Fr}=u / c$, which describes the ratio between the two velocity scales. We are interested into perturbations of stationary solutions. These solutions are balance between pressure force and source term: $\partial_{x} p=-g h \Theta \partial_{x} z$. Hereafter we consider the following three families of equilibria, with zero velocity:

$$
\left\{\begin{array} { l } 
{ u = 0 , } \\
{ \Theta = \mathrm { cst } , } \\
{ h + z = \mathrm { cst } , }
\end{array} \quad \left\{\begin{array} { l } 
{ u = 0 , } \\
{ z = \mathrm { cst } , } \\
{ \Theta \frac { h ^ { 2 } } { 2 } = \mathrm { cst } , }
\end{array} \quad \left\{\begin{array}{l}
u=0, \\
h=\mathrm{cst}, \\
z+\frac{h}{2} \ln (\Theta)=\mathrm{cst} .
\end{array}\right.\right.\right.
$$

The first aim is to write a scheme that preserves these steady states. Indeed, if the scheme does not preserve the steady states, spurious velocity and pressure modes would appear and would destroy the accuracy of the scheme for small velocity (low Froude regime). The second aim is to capture the dynamics near an equilibrium with an acceptable cost. For example, we consider the following perturbation $O(\mathrm{Fr})$ of the first steady equilibria with $\mathrm{Fr} \ll 1$. In that case, the perturbation has a small amplitude but moves with a large propagation speed of order $O(1 / \mathrm{Fr})$. Therefore, implicit schemes are usually required to filter these small fast waves.

\section{Two-speed relaxation system}

To simplify the implicit treatment of the dynamics, we propose a relaxation model that linearizes the fast scale associated to the gravity waves. We introduce two additional unknows $\Pi(x, t)$ and $v(x, t)$ and consider the following extended hyperbolic system

$$
\left\{\begin{array}{l}
\partial_{t} h+\partial_{x}(h v)=0, \\
\partial_{t}(h u)+\partial_{x}(h u v+\Pi)=-g h \partial_{x} z, \\
\partial_{t}(h \Theta)+\partial_{x}(h \Theta v)=0, \\
\partial_{t} \Pi+v \partial_{x} \Pi+h_{m} \lambda^{2} \partial_{x} v=\frac{1}{\varepsilon}(p(h, \Theta)-\Pi) \\
\partial_{t} v+v \partial_{x} v+\frac{1}{h_{m}} \partial_{x} \Pi=-\frac{h}{h_{m}} g \Theta \partial_{x} z+\frac{1}{\varepsilon}(v-u)
\end{array}\right.
$$

with $h_{m}>0$ and $\lambda>0$ are constant relaxation parameters and where $\varepsilon>0$ is the relaxation parameter. This system is an approximation of (1) in the limit $\varepsilon$ tends to zero. This result can be shown formally.

Proposition 1 As $\varepsilon \rightarrow 0$, the relaxation system (3) is consistent at first order in $\varepsilon$ with 


$$
\left\{\begin{array}{l}
\partial_{t} h+\partial_{x}(h u)=\varepsilon \partial_{x}\left(\beta\left(\partial_{x} p+g h \partial_{x} z\right)\right), \\
\partial_{t}(h u)+\partial_{x}\left(h u^{2}+\Theta \frac{g}{2} h^{2}\right)=-h g \Theta \partial_{x} z+\varepsilon \partial_{x}\left(u \beta\left(\partial_{x} p+g h \partial_{x} z\right)\right)+\varepsilon \partial_{x}\left(\gamma \partial_{x} u\right) \\
\partial_{t}(h \Theta)+\partial_{x}(h \Theta u)=\varepsilon \partial_{x}\left(\Theta \beta\left(\partial_{x} p+g h \partial_{x} z\right)\right),
\end{array}\right.
$$

with $\beta=\left(\frac{h}{h_{m}}-1\right), \gamma=\left(h_{m} \lambda^{2}-h c^{2}\right)$.

The proof is based on a classical Chapman-Enskog expansion detailed for the Euler system case in [5]. Note that an equilibrium given by $\partial_{x} p=-g h \Theta \partial_{x} z$ and $u=0$ is still a steady state of the first order approximation (4) of the relaxation system. It is not the case for all the relaxation models like the Jin-Xin relaxation used in [3]. This property is necessary, but not sufficient, to obtain a well-balanced scheme since we will discretize the relaxation system (3) and not the original one. In [5], the entropy stability of (4) is analysed. This computation adapted to our model gives the following stability conditions: $\beta \geq 0, \gamma \geq 0$.

\section{Semi-implicit scheme}

The structure of the relaxation system (3) enables us to devise a semi-implicit scheme with a simple implicit part. This scheme is based on a splitting method between the different time-scale dynamics contained in (3). We split system (3) into the convection part $(\mathrm{C})$, the gravity waves part $(\mathrm{W})$ and the relaxation part $(\mathrm{R})$ :

$$
\begin{aligned}
& \text { (C) }\left\{\begin{array}{l}
\partial_{t} h+\partial_{x}(h v)=0, \\
\partial_{t}(h u)+\partial_{x}\left(h u v+\mathcal{F}^{2} \Pi\right)=-\mathcal{F}^{2} g h \Theta \partial_{x} z, \\
\partial_{t}(h \Theta)+\partial_{x}(h \Theta v)=0, \\
\partial_{t} \Pi+v \partial_{x} \Pi+h_{m} \lambda^{2} \partial_{x} v=0 \\
\partial_{t} v+v \partial_{x} v+\frac{\mathcal{F}^{2}}{h_{m}} \partial_{x} \Pi=-\mathcal{F}^{2} \frac{h}{h_{m}} g \Theta \partial_{x} z
\end{array}\right. \\
& \left(\begin{array}{l}
\partial_{t} h=0, \\
\partial_{t}(h u)+\left(1-\mathcal{F}^{2}\right)\left(\partial_{x} \Pi+h g \partial_{x} z\right)=0, \\
\partial_{t} h \Theta=0 \\
\partial_{t} \Pi+\left(1-\mathcal{F}^{2}\right) h_{m} \lambda^{2} \partial_{x} v=0 \\
\partial_{t} v+\left(1-\mathcal{F}^{2}\right)\left(\frac{1}{h_{m}} \partial_{x} \Pi+\frac{h}{h_{m}} g \partial_{x} z\right)=0
\end{array}\right. \\
& (R) \quad\left\{\begin{array}{l}
\partial_{t} \Pi=\frac{1}{\varepsilon}(p(h, \Theta)-\Pi), \quad \partial_{t} v=\frac{1}{\varepsilon}(u-v),
\end{array}\right.
\end{aligned}
$$

where $\mathcal{F}=\max \left(\mathcal{F}_{\min }, \min \left(\frac{u}{\sqrt{h \Theta g}}, 1\right)\right)$ is an estimation of the global Froude number. Introduced in [4], this dynamic splitting allows to adapt itself to the dynamics.

In practice, the relaxation step $(\mathrm{R})$ is treated as a projection: $\Pi=p(h, \Theta)$ and $v=u$. The key point is the discretization of the waves part (W) with an implicit solver. First, we note that $\partial_{t} h=0$ so that the topography source term can be treated explicitly. The construction of the implicit scheme is based on the following remark: the equations on $v$ and $\Pi$ form a linear independent system. We can thus discretize 
all the equations of (W) with an implicit Euler scheme and then we get an implicit elliptic problem on $\Pi^{n+1}$ by plugging the expression of $v^{n+1}$ in the $\Pi$ equation. This elliptic problem is linear and has constant coefficient. After solving this problem, we obtain $\Pi^{n+1}$ and then $v^{n+1}$ and $(h u)^{n+1}$ can be computed with an explicit cost. For the spatial discretization, we use a classical finite volume scheme for the elliptic part and the central flux for the first derivative. The final algorithm writes:

- Step 1: solve

$$
\begin{aligned}
& \left(\Pi_{j}^{n+1}-\left(1-\mathcal{F}^{2}\right)^{2} \Delta t^{2} \lambda^{2} \frac{\Pi_{j+1}^{n+1}-2 \Pi_{j}^{n+1}+\Pi_{j-1}^{n+1}}{\Delta x^{2}}\right)= \\
& \quad \Pi_{j}^{n}-\Delta t\left(1-\mathcal{F}^{2}\right) \lambda^{2} \frac{v_{j+1}^{n}-v_{j-1}^{n}}{2 \Delta x}\left(1-\mathcal{F}^{2}\right)^{2} \Delta t^{2} \lambda^{2} \frac{1}{\Delta x}\left(S_{j+\frac{1}{2}}^{n}-S_{j-\frac{1}{2}}^{n}\right),
\end{aligned}
$$

with

$$
S_{j+\frac{1}{2}}^{n}=h_{j+\frac{1}{2}}^{n} \Theta_{j+\frac{1}{2}}^{n} \frac{z_{j+1}-z_{j}}{\Delta x},
$$

where the quantities $h_{j+\frac{1}{2}}$ and $\Theta_{j+\frac{1}{2}}$ will be define below.

- Step 2: compute

$$
\begin{gathered}
v_{j}^{n+1}=v_{j}^{n}-\left(1-\mathcal{F}^{2}\right) \frac{\Delta t}{h_{m}} \frac{\Pi_{j+1}^{n+1}-\Pi_{j-1}^{n+1}}{2 \Delta x}-\left(1-\mathcal{F}^{2}\right) \frac{\Delta t}{h_{m}} \frac{g}{2}\left(S_{j+\frac{1}{2}}^{n}-S_{j-\frac{1}{2}}^{n}\right), \\
(h u)_{j}^{n+1}=(h u)_{j}^{n}-\Delta t\left(1-\mathcal{F}^{2}\right) \frac{\Pi_{j+1}^{n+1}-\Pi_{j-1}^{n+1}}{2 \Delta x}-\frac{g \Delta t}{2}\left(1-\mathcal{F}^{2}\right)\left(S_{j+\frac{1}{2}}^{n}-S_{j-\frac{1}{2}}^{n}\right) .
\end{gathered}
$$

\section{Well-balanced fluxes}

The convective part (C) is discretized with a first order explicit finite volume scheme. The numerical flux for the transport terms is constructed so that it preserves the steady states and this will provide the value of $h_{j+\frac{1}{2}}$ and $\Theta_{j+\frac{1}{2}}$. Following [1,6], the idea consists in splitting the flux and then using a specific numerical fluxes for each part. Here, we propose to decompose the flux term in three parts:

$$
\begin{aligned}
\left(\begin{array}{c}
\partial_{x}(h v), \\
\partial_{x}\left(h u v+\mathcal{F}^{2} \Pi\right) \\
\partial_{x}(h \Theta v) \\
v \partial_{x} \Pi+h_{m} \lambda^{2} \partial_{x} v \\
v \partial_{x} v+\frac{\mathcal{F}^{2}}{h_{m}} \partial_{x} \Pi
\end{array}\right) & =\partial_{x}\left(\begin{array}{c}
h v \\
h u v+\mathcal{F}^{2} \Pi \\
h \Theta v \\
0 \\
0
\end{array}\right)+\left(\begin{array}{c}
0 \\
0 \\
0 \\
v \partial_{x} \Pi \\
v \partial_{x} v
\end{array}\right)+\partial_{x}\left(\begin{array}{c}
0 \\
0 \\
0 \\
h_{m} \lambda_{c} v \\
\frac{1}{h_{m}} \mathcal{F}^{2} \Pi
\end{array}\right) \\
& =\partial_{x} F_{c}+F_{n c}^{*}+\partial_{x} F_{\ell a}
\end{aligned}
$$

For the non-conservative part $F_{n c}^{*}$, we use a non-conservative upwind scheme. For the linear acoustic part $F_{\ell a}$, we propose a modified acoustic flux that takes into account spatial variation due to the source term using the Jin-Levermore method [7]. 
The linear acoustic part writes:

$$
\partial_{t} \Pi+h_{m} \lambda^{2} \partial_{x} v=0, \quad \partial_{t} v+\mathcal{F}^{2} \frac{1}{h_{m}} \partial_{x} \Pi=0,
$$

and can be diagonalized as follows:

$$
\left\{\begin{array}{l}
\partial_{t}\left(\frac{h_{m} \lambda}{\mathcal{F}} v-\Pi\right)-\mathcal{F} \lambda \partial_{x}\left(\frac{h_{m} \lambda}{\mathcal{F}} v-\Pi\right)=0 \\
\partial_{t}\left(\frac{h_{m} \lambda}{\mathcal{F}} v+\Pi\right)+\mathcal{F} \lambda \partial_{x}\left(\frac{h_{m} \lambda}{\mathcal{F}} v+\Pi\right)=0
\end{array}\right.
$$

To define the intermediate value of $\Pi$ and $v$ in the fluxes at node $x_{j+\frac{1}{2}}$, we thus consider the upwinded quantities:

$$
\left\{\begin{array}{l}
\left(\frac{h_{m} \lambda v}{\mathcal{F}}-\Pi\right)_{j+\frac{1}{2}}=\frac{h_{m} \lambda}{\mathcal{F}} v\left(x_{j+\frac{1}{2}}^{+}\right)-\Pi\left(x_{j+\frac{1}{2}}^{+}\right) \\
\left(\frac{h_{m} \lambda v}{\mathcal{F}}+\Pi\right)_{j+\frac{1}{2}}=\frac{h_{m} \lambda}{\mathcal{F}} v\left(x_{j+\frac{1}{2}}^{-}\right)+\Pi\left(x_{j+\frac{1}{2}}^{-}\right) .
\end{array}\right.
$$

Following [7] and as already done for the Euler gravity system in [2], we precise these formula by considering the possible spatial variation of $\Pi$ at equilibria:

$$
\Pi\left(x_{j}\right) \approx \Pi\left(x_{j+\frac{1}{2}}^{-}\right)-\frac{\Delta x}{2} \partial_{x} \Pi\left(x_{j+\frac{1}{2}}\right) \approx \Pi\left(x_{j+\frac{1}{2}}^{-}\right)+\frac{\Delta x}{2} h\left(x_{j+\frac{1}{2}}\right) \Theta\left(x_{j+\frac{1}{2}}\right) g \partial_{x} z\left(x_{j+\frac{1}{2}}\right) .
$$

We define similarly $\Pi\left(x_{j+\frac{1}{2}}^{+}\right)$. Then plugging these values in (6) and considering $v\left(x_{j+\frac{1}{2}}^{+}\right)=v\left(x_{j+1}\right)$ and $v\left(x_{j+\frac{1}{2}}^{-}\right)=v\left(x_{j}\right)$, we obtain at the discrete level:

$$
\left\{\begin{array}{l}
\left(\frac{h_{m} \lambda v}{\mathcal{F}}-\Pi\right)_{j+\frac{1}{2}}=\frac{h_{m} \lambda}{\mathcal{F}} v_{j+1}-\Pi_{j+1}+\frac{\Delta x}{2} h_{j+\frac{1}{2}} \Theta_{j+\frac{1}{2}} g \frac{z_{j+1}-z_{j}}{\Delta x} \\
\left(\frac{h_{m} \lambda v}{\mathcal{F}}+\Pi\right)_{j+\frac{1}{2}}=\frac{h_{m} \lambda}{\mathcal{F}} v_{j}+\Pi_{j}-\frac{\Delta x}{2} h_{j+\frac{1}{2}} \Theta_{j+\frac{1}{2}} g \frac{z_{j+1}-z_{j}}{\Delta x}
\end{array}\right.
$$

Then we obtain the following intermediate values for the modified acoustic fluxes:

$$
\left\{\begin{array}{l}
v_{j+\frac{1}{2}}^{*}=\frac{1}{2}\left(v_{j+1}+v_{j}\right)-\frac{\mathcal{F}}{2 h_{m} \lambda_{c}}\left(\Pi_{j+1}-\Pi_{j}+g h_{j+\frac{1}{2}} \Theta_{j+\frac{1}{2}}\left(z_{j+1}-z_{j}\right)\right), \\
\Pi_{j+\frac{1}{2}}^{*}=\frac{1}{2}\left(\Pi_{j+1}+\Pi_{j}\right)-\frac{h_{m} \lambda_{c}}{2 \mathcal{F}}\left(v_{j+1}-v_{j}\right) .
\end{array}\right.
$$

Finally, for the convection part, we use an upwind scheme at the velocity $v_{j+\frac{1}{2}}^{*}$ for the quantities $h, h u, h \Theta$ and we use $\Pi_{j+\frac{1}{2}}^{*}$ for the pressure term.

To ensure the well-balanced property, we consider the following discretization for the source terms [2]:

$$
S_{j}=-g \frac{1}{2}\left(S_{j+\frac{1}{2}}+S_{j-\frac{1}{2}}\right)
$$

where $S_{j+\frac{1}{2}}$ are defined in (5). 
Proposition 2 Considering that $u_{j}=0, v_{j}=0$ and $\Pi_{j}=\frac{1}{2} g \Theta_{j} h_{j}^{2}$, the schemes for the convective part $(C)$ and for the wave part $(W)$ are well-balanced for the three type of steady states:

$$
\left\{\begin{array} { l } 
{ \Theta _ { j } = c s t , } \\
{ h _ { j } + z _ { j } = c s t , }
\end{array} \quad \left\{\begin{array} { l } 
{ z _ { j } = c s t , } \\
{ \Theta _ { j } ( h _ { j } ) ^ { 2 } / 2 = c s t , }
\end{array} \quad \left\{\begin{array}{l}
h_{j}=c s t \\
z_{j}+h_{j} \ln \left(\Theta_{j}\right)=c s t
\end{array}\right.\right.\right.
$$

if we choose in the scheme

$$
h_{j+\frac{1}{2}}=\frac{1}{2}\left(h_{j}+h_{j+1}\right), \quad \Theta_{j+\frac{1}{2}}= \begin{cases}\frac{\Theta_{j+1}-\Theta_{j}}{\ln \left(\Theta_{j+1}\right)-\ln \left(\Theta_{j}\right)}, & \text { if } \Theta_{j+1} \neq \Theta_{j}, \\ \Theta_{j}, & \text { if } \Theta_{j+1}=\Theta_{j} .\end{cases}
$$

Indeed, with this choice, then $v_{j+\frac{1}{2}}^{*}=0$ for all the steady-states. Consequently, all the transport terms at velocity $v_{j+\frac{1}{2}}^{*}$ in $(C)$ vanish. It remains to prove that the balance between the pressure term $\left(\Pi_{j+\frac{1}{2}}^{*}-\Pi_{j-\frac{1}{2}}^{*}\right) / \Delta x=\left(\Pi_{j+1}^{n}-\Pi_{j-1}^{n}\right) /(2 \Delta x)$ and the soure term. This follows from the choice (8). We do not detail the computation here. For the implicit scheme for $(W)$, the preservation of these steady states results from this balance between discret gradient pressure and the source term and also from the centered discretization.

\section{Numerical results}

First, we consider a classical test case for well-balanced schemes. The initial data is taken as a steady state and we compare the classical Rusonov scheme with the semi-implicit two-speed well-balanced scheme (SI two-speed WB) on a large time interval $\left[0, T_{f}\right]$. The CFL condition of our scheme is given by

$$
\Delta t \leq \frac{\Delta x}{\max _{x}|u(t, x)+\mathcal{F}(t) \sqrt{h(t, x) \Theta(t, x) g}|} .
$$

The parameter $\mathcal{F}(t)$ depends on an important parameter $\mathcal{F}_{\text {min }}$. If $u=0$, there is still a small part of the gravity wave dynamics which is explicit and generates a CFL condition: $\Delta t \leq \Delta x /\left(\mathcal{F}_{\min } \sqrt{h \Theta g}\right)$. Hence, decreasing this parameter $\mathcal{F}_{\text {min }}$ increases the time step. We remark also that when $\mathcal{F}_{\min }=1$, the scheme is explicit. The initial data are given by $u_{0}(x)=v_{0}(x)=0, \Pi_{0}(x)=\frac{1}{2} \Theta_{0}(x) h_{0}(x)^{2}$ with the following topography $z(x)$, initial water height $h_{0}(x)$ and temperature $\Theta_{0}(x)$ :

$(S T 1)$

$(S T 3)$

$$
\begin{array}{llrl}
z(x)=0.1+G_{x_{0}, \sigma}(x), & h_{0}(x)=8.0-z(x), & \Theta_{0}(x)=1, \\
z(x)=1, & h_{0}(x)=1.0+0.2 G_{x_{0}, \sigma}(x), \Theta_{0}(x)=\frac{1}{g h_{0}(x)^{2}}, \\
z(x)=x(1-x), & h_{0}(x)=1, & \Theta_{0}(x)=2 e^{-x(1-x)}
\end{array}
$$


with $G(x, \sigma)=\frac{1}{\sqrt{2 \pi \sigma}} \exp \left(-\frac{\left(x-x_{0}\right)^{2}}{\sigma}\right)$ and $\sigma=0.06$. We consider $g=1, N_{c}=200$ cells and $T_{f}=20$. The results are given in the table below.

The results show that the SI two-speed WB is exactly well-balanced in the explicit version $\left(\mathcal{F}_{\min }=1\right)$. In the semi-implicit case, it is more complicated. At the theoretical level the scheme is well-balanced, but the implicit part generates very small errors close to machine precision and these errors are slowly propagated. The scheme does not preserve the steady states exactly. However, we remark that with $\mathcal{F}_{\min }=0.005$, the errors are very small (between $1.0 E^{-11}$ and $1.0 E^{-13}$ ) with a time step 200 larger than the one of the explicit schemes. In the following, we will show that the scheme well capture the flow around steady states and that these pertubative errors does not deteriorate the results contrary to classical non well-balanced schemes.

\begin{tabular}{|c|c|c|c|c|c|}
\hline$\Delta t$ /Error & Tests & $\begin{array}{c}\text { Explicit } \\
\text { Rusanov }\end{array}$ & $\begin{array}{c}\text { SI two-speed WB } \\
\left(\mathcal{F}_{\min }=1\right)\end{array}$ & $\begin{array}{c}\text { I two-speed WB } \\
\left(\mathcal{F}_{\min }=0.1\right)\end{array}$ & $\begin{array}{c}\text { SI two-speed WB } \\
\left(\mathcal{F}_{\min }=0.005\right)\end{array}$ \\
\hline \multirow{4}{*}{ ST1 } & Error $h$ & $1.5 E^{-2}$ & $1.5 E^{-17}$ & $1.5 E^{-13}$ & $3.6 E^{-13}$ \\
\cline { 2 - 7 } & Error $u$ & $5.9 E^{-3}$ & $1.5 E^{-15}$ & $4.8 E^{-11}$ & $6.7 E^{-13}$ \\
\cline { 2 - 7 } & Error $\Theta$ & 0.0 & 0.0 & 0.0 & 0.0 \\
\cline { 2 - 7 } & $\Delta t$ & $8.1 E^{-4}$ & $7.1 E^{-4}$ & $7.1 E^{-3}$ & $1.42 E^{-1}$ \\
\hline \multirow{5}{*}{ ST2 } & Error $h$ & $9.3 E^{-2}$ & 0.0 & $6.4 E^{-11}$ & $8.4 E^{-12}$ \\
\cline { 2 - 6 } & Error $u$ & $7.3 E^{-9}$ & 0.0 & $8.7 E^{-13}$ & $1.3 E^{-13}$ \\
\cline { 2 - 6 } & Error $\Theta$ & 0.13 & $1.8 E^{-11}$ & $8.2 E^{-11}$ & $6.0 E^{-12}$ \\
\cline { 2 - 6 } & $\Delta t$ & $2.5 E^{-3}$ & $2.3 E^{-3}$ & $2.3 E^{-2}$ & $4.7 E^{-1}$ \\
\hline \multirow{4}{*}{ ST3 } & Error $h$ & 0.59 & 0.0 & $7.1 E^{-9}$ & $1.38 E^{-12}$ \\
\cline { 2 - 6 } & Error $u$ & 0.65 & $1.6 E^{-15}$ & $1.0 E^{-9}$ & $4.4 E^{-14}$ \\
\cline { 2 - 6 } & Error $\Theta$ & 0.19 & 0.0 & $9.4 E^{-9}$ & $1.4 E^{-12}$ \\
\cline { 2 - 6 } & $\Delta t$ & $2.4 E^{-3}$ & $1.8 E^{-3}$ & $1.8 E^{-2}$ & 0.49 \\
\hline
\end{tabular}

Then we introduce a perturbation in the (ST3) test-case, $h_{0}(x)=1+0.001 G_{0.8, \sigma}(x)$, on the domain is [0,3]. In Figure (1), we compare the explicit Rusanov scheme with the SI two-speed WB scheme. We observe that the non-WB explicit Rusanov scheme is not able to capture the physical perturbation: the scheme creates a numerical perturbation larger that the physical one (in red), for 1200 cells, or with the same size (in blue) with 12000 cells. The SI two-speed WB scheme does not create numerical perturbations and capture correctly the physical perturbation with a coarser grid. The results are better with 600 cells and the SI two-speed WB scheme that with 12000 cells with the non-WB Rusanov scheme. Additionally, the results obtained with the SI two-speed WB scheme are given with a 10 times larger time step than the explicit one. We cannot increase too much the time step here since the implicit part of the schemes would create numerical diffusion on the gravity wave part. 

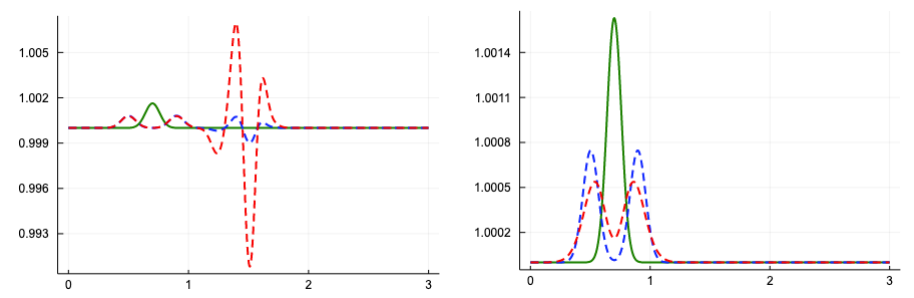

Fig. 1 Left: explicit Rusanov scheme; In green the initial data. In red the solution on a semi-coarse grid (1200 cells), in blue the solution on a fine grid (12000 cells). Right: SI two-speed WB; in green the initial data. In red the solution on a coarse grid ( 600 cells), in blue the solution on a semi-coarse grid (4800 cells).

\section{Conclusion}

In this paper, we propose a semi-implicit relaxation scheme for the Ripa model, that is well-adapted to treat the low-Froude regime. Indeed, we are able to take very large time step compared to the gravity waves time scale and the accuracy of the scheme is independent of the Froude number. The two-speed relaxation allows to have an implicit step with a constant linear Laplacian. Additionally the scheme is able to preserve non-trivial steady states with a very good accuracy, with small errors for very large time steps. However the scheme is not able to treat wet/dry transitions. The 2D extension have been performed for the Euler system in [5]. In the future, we propose to extend the method to flows around equilibria MHD system.

\section{References}

1. P. Tiam Kapen, T. Ghislain, A New Flux Splitting Scheme Based on Toro-Vazquez and HLL Schemes for the Euler Equations, Journal of Computational Methods in Physics. 2018

2. E. Franck, L. Mendoza, Finite Volume Scheme with Local High Order Discretization of the Hydrostatic Equilibrium for the Euler Equations with External Forces, Journal of Scientific Computing, October 2016, Volume 69, Issue 1.

3. D. Coulette, E. Franck, P. Helluy, A. Ratnani, E. Sonnendrueker, Implicit time schemes for compressible fluid models based on relaxation methods, Computers and Fluids Volume 188, 30 June 2019, Pages 70-85.

4. D. Iampietro, F. Daube, P. Galon, J. M. Herard, A Mach-Sensitive Implicit-Explicit Scheme Adapted to Compressible Multi-scale Flows Journal of Computational and Applied Mathematics Volume 340, 1 October 2018, Pages 122-150.

5. F. Bouchut, E. Franck, L. Navoret, , preprint 2019.

6. E. F. Toro, M. E. Vaquez-Cendon, Flux splitting schemes for the Euler equations, Computers and Fluids, vol. 70, 2012

7. S. Jin, D. Levermore, Numerical Schemes for Hyperbolic Conservation Laws with Stiff Relaxation Terms, J. Computational Physics 126 (1996), 449-467.

8. C. Berthon, V. Desveaux, C. Klingenberg, Well-balanced scheme to capture non explicit steady-states: Ripa model Mathematics of Computation, Volume 85, Number 300, July 2016. 